surface. As the tissues in this part are well nourished, first-intention is the rule.

This operation renders direct surgical attack feasible in many rebellious forms of seminal vesicular disease which have formerly been left unaided, or which have responded unsatisfactorily to palliative measures of treatment. Acting on this assumption $I$ have in this manner opened and drained thickened and catarrhallydistended seminal vesicles, have freed them from dense inflammatory adhesions, have removed a neoplasm from the interior of one of them, and have entirely extirpated one. Aside from extirpation of the seminal vesicles, much of the surgery $I$ am now doing in this connection is in the nature of pioneer work. As the patients on whom I have practiced it are, however, still in the hospital, convalescent, or have been only recently discharged, it is as yet too early to draw conclusions as to its true surgical value. I have accordingly called attention in the title of my paper to the fact that my report is a preliminary one. This specification it is needless to say does not apply to my method of operating, the advantages of which are established. The operation, as I have stated, exposes the prostate most satisfactorily, making its removal comparatively easy. Although I have removed a very large number of pros-

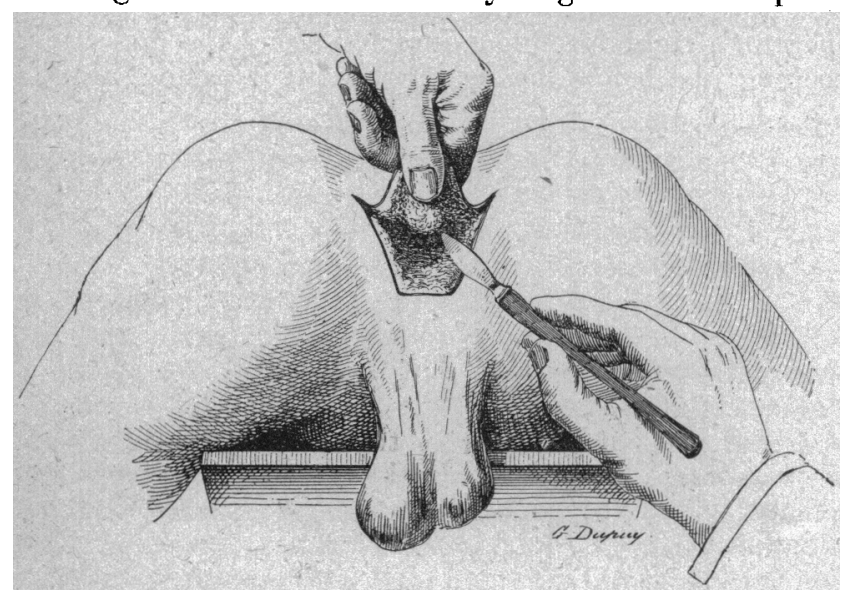

Fig. 3.

tates from those suffering from the effects of prostatic hypertrophy, I have almost uniformly done so in connection with a suprapubic or perineal cystotomy, and shall probably continue to do so in spite of the easy route afforded by the operation just described, since I consider drainage and treatment of the bladder in that class of cases a most important feature in the aftertreatment, the accomplishment of which is impossible in connection with an extravesical operation. For suppurative conditions in connection with the prostate the operation I have described would be of practical value.

Up to the present time I have employed my operative method on five occasions. The patients have all recovered most satisfactorily, their stay in the hospital averaging about three weeks. In three of them the purpose of the operation was to expose chronically diseased seminal vesicles for incision and drainage. In these instances free longitudinal incisions into the diseased sacs were made and, were the interior of the organs was found to be granular from chronic catarrh. such tissue was removed by gentle curettage. In all cases the cavities of the sacs were left packed with gauze, while gauze drainage to the surface of the wound was established. Wherever the sacs were found bound down by perivesicular inflammatory adhesions, such were broken up, leaving the organs free. This is an original method of treating chronic seminal vesiculitis. I think it will prove of much value in connection with very aggravated cases. As my experience with it is as yet, however, very recent, I will not at this time comment further on it.

One of my cases represented an instance of extirpation of a seminal vesicle, while from another I removed a neoplasm from the interior of a seminal vesicle, leaving the sac intact, the first operation of its kind on record. The neoplasm in question was an adenoma having, however, associated with it certain evidences which made the examining pathologist fearful of the coexistence of carcinoma.

\section{MYASTHENIA GRAVIS PSEUDOPARALYTICA.}

(ASTHENIC BULBAR PARAIYSIS.)*

J. R. BUIST, M.D., AND E. G. WOOD, M.D.

NASHVILLE, TENN.

In 187\%, Wilks, of Guy's Hospital, London, published the report of a case of "Bulbar Paralysis without Anatomical Changes." "The patient, a girl, could scarcely move about, spoke slowly and had strabismus. For a month she remained in much the same condition, then all the symptoms became aggravated and in three days they assumed the characters of bulbar paralysis. There were indistinctness of speech, difficulty of swallowing and inability to cough. Respiration became increasingly difficult, and death rapidly supervened." The next year Erb reported three cases in which the chief symptoms were a striking paresis of muscles, especially of mastication, of the neck and the eves (plosis), less of those of deglutition, the tongue and extremities.

During the twenty years that have passed since Wilks reported this interesting condition, 62 cases have been reported, including our own, most of them (46) from the continent of Europe-10 from England and 6 from this country. Though there has been a notable lack of unanimity in the nomenclature of this affection, the clinical features of the cases recorded by the various observers have been so strikingly similar in all their salient features, and the results of the autopsies have, with one or two doubtful exceptions, so uniformly presented a complete absence of all signs of organic disease both macroscopic and microscopic that there can be no reasonable doubt that practically all the cases belong to the same category.

To the earliest observers the symptoms which attracted the greatest attention were those referred to the muscles innervated by the bulbar nuclei, hence whatever the lesion or disturbance of function might prove to be, it was naturally referred to the bulb and the various designations of "bulbar paralysis without anatomical changes" (Wilks, 187\%) and "asthenic bulbar paralysis" (Strümpell, Church, Dana, and Collins, in "Twentieth Century Practice") were given to the disease.

In 1890 , Shaw ${ }^{2}$ reported a characteristic example of the disease. In this case he drew particular attention to the weakness and exhaustibility not only in the muscles innervated by the bulb, but also in the muscles of the eyes and extremities. During recent years this weakness and exhaustibility of the muscles generally has taken perhaps a more important place in the symptomatology of the disease than that formerly given to the bulbar symptoms.

In 1895 , Jolly ${ }^{8}$ reported a case under the title of "Myasthenia Gravis Pseudoparalytica," and he seems

* Read before the Nashville Academy of Medicine, Dec. 11. 1900. 
to have been the first to use this name. Recently the Finglish writers, notably Buzzard, with Sanger Brown in this country, have adopted the same title. While it may be claimed that such a title is unscientific, one may urge that it is not misleading, that it suggests no pathologic conditions that might prove to be erroneous, and that it refers especially to the characteristic clinical feature of the disease.

Etiology.-As to the etiology, practically nothing is known. Sex seems to play no important part. It has been observed most frequently in young adults. A few cases have been preceded by influenza. typhoid fever, prolonged worry or mental or physical exertion, but in the great majority of the cases the patients have been strong healthy individuals, free from any apparent taint, hereditary or acquired, before the attack, and in no instance recorded has any observer been able to attribute the disease to any definite cause. Rarely has there been a family tendency to nervous disease. Syphilis and alcohol seem to play no part as an etiologic factor.

Pathology.-At present nothing definite is known as to the true nature of the disease. In seventeen instances autopsies have been held by competent observers, with the practically uniform result that no lesions have been discovered either in the affected muscles or in any part of the nervous system, central or peripheral, that were sufficient to account for the symptoms. Careful and extensive microscopic examination of the whole nervous system by the most modern methods failed to discover any departure from the normal in 11 of the 17 autopsies; in the other 6 the lesions were slight and inconstant, arid consisted of slight degenerative changes in the intramedullary portion of the anterior roots in one case, in the cells of the motor gray matter from the oculomotor nucleus through the whole length of the cord in another, of partial disappearance of the chromatophilic elements of the third, sixth, seventh and twelfth nuclei in another, and of slight medullary hemorrhages that possibly occurred while dying in the other three. By some the disease is considered to be toxic, the result of disturbed metabolism, and it is possible that the poison produces changes in the cells which can not be observed under ordinary modes of investigation, but which may in the future be demonstrated by more recent methods of examination.

An interesting experiment by Dr. Farquhar Buzzard ${ }^{4}$ goes a long way to prove that the disease is one of the nervous system and not a myopathy. In a case under his observation he completely exhausted the biceps muscle, by faradic stimulation until movements were no Icnger visible; he then immediately applied galvanism and a prompt and vigorous contraction occurred. This tends strongly to show that the exhaustion was not in the muscle but in the conducting power of the nerve. The absence of muscular atrophy also militates against the myopathic nature of the disease.

Symptoms. - The characteristic features of the disease are the weakness of the muscles and the rapidity with which they become exhausted, the tendency to affect not only the bulbar muscles but also those of the eyes and extremities, the occurrence of remissions and exacerbations with a tendency to vary from day to day, the presence of the myasthenic reaction, the retention of intellectual and sexual power, and the absence of atrophy and fibrillary twitching, of sensory disturbance, of reaction of degeneration, of bladder or rectal troubles, or of interference with the reflexes. The onset is usually gradual, but sometimes rapid. Though the weakness may begin in any muscle, the cranial nerve muscles usually suffer first, and in the majority of cases the earliest symptom is either ptosis or diplopia; for this reason many of these patients first consult the oculist. The ptosis or diplopia may be slight, may be intermittent or may disappear altogether for an indefinite period, to recur again in a marked degree; after a time it usually becomes a permanent symptom which varies, however, from day to day, and tends to become worse in the evenings. The liability to fatigue of the levator palpebræ is readily shown if the patient looks steadily upward at an object held above his eyes, when the lids are seen to gradually droop until the eyes are nearly closed. There may be weakness of any or all of the ocular muscles, resulting in complete ophthalmoplegia.

Suckling ${ }^{5}$ published two characteristic examples under the title: "Functional Ophthalmoplegia with General Paralysis and Implication of Cranial Nerves in Young Women." In these cases there was a great tendency to osciliatory movements of the eyeballs when fixed for a time to their limit in the direction of the weak muscle. In some instances the patients can not raise their eyebrows, wrinkle the forehead nor close the eyes tightly; in others the weakness of the lower facial muscles is shown by a calm, rather expressioniless countenance. Sooner or later the muscles innervated by the bulbar nuclei are affected; weakness in these muscles may be the earliest symptom, or it may not appear until near the end of the case. It is owing to the prominence of these bulbar symptoms that the disease has been designated "asthenic bulbar paralysis." All the symptoms of true bulbar paralysis may be present, but they are usually less marked, nearly always bilateral and are characterized also by exhaustibility and variability from day to day. There is difficulty in biting, mastication, deglutition and articulation; the palate reflex may be weak and on phonation the soft palate but slightly elevated on one or both sides; there may be inability to protrude the tongue beyond the teeth, or if projected to its normal extent it soon tires, begins to quiver and recedes into the mouth; difficulty in sucking, blowing or inflating the cheeks may be observed. In many cases the patient begins his meal all right, but in a few minutes, though still hungry, he is compelled to rest owing to the weakness in his muscles of mastication, or before he has satisfied his hunger he is no longer able to swallow and fluids regurgitate through his nose. The difficulty in articulation is shown by the weakness of the voice after use. At first the patient can speak clearly and distinctly, but his voice gradually grows weaker until he can no longer articulate; after a short rest he can proceed as before. The disease is prone to affect the respiratory muscles, producing attacks of dyspnea of an alarming character; indeed, the majority of the deaths have been due to asphyxia from this cause. The rapid fatigue of the respiratory muscles is well shown in our patient, who lost three-quarters of an inch in expansive movement after eight or ten inspirations.

The muscles of the head and neck may be affected with a tendency of the head to fall backward. The extremities are weak, the patient may be unable to raise his arms above his head, or, if he can do so, he can not maintain them in this position beyond a few minutes when they gradually fall to his side, but after a brief rest he may be able to raise them as high as before. His grip is feeble. In writing he begins rapidly, but after a few lines it becomes slower and labored until he is compelled to stop. The patient may be able to walk well for a short distance, then his gait becomes 
feeble and irregular until he is forced to sit down and rest; he may be unable to lift one knee over the other. In some cases the weakness is so marked that the patient is unable to raise himself out of bed or to rise from a chair.

In addition to the weakness and exhaustibility, with in some cases a permanent paresis, which may affect any of the voluntary muscles, other striking features of the disease are its tendency to be aggravated in the evening, especially after exercise, the occasional exaggeration of the weakness when the patient's attention is drawn to his condition and the better performance of involuntary than voluntary movements. Remissions and exacerbations are common and the patient may for weeks, months, or even years-five years in Dreschfeld's ${ }^{9}$ case-appear to have made a complete recovery and then, perhaps suddenly, there is a recurrence of all the symptoms.

Myasthenic Reaction.-The electrical reactions in the affected muscles are interesting. If the muscle is not fatigued by voluntary exertion there is a prompt and good response to faradic stimulation-the vigor of the contraction, however, varies in different muscles and in the same one at different times. As the applications of the current are rapidly repeated, the movements gradually decline in extent and force until in a few minutes contractions are no longer visible, but after a minute's rest vigorous contractions are again induced by faradism. This peculiar exhaustibility of the muscles to the faradic current is called the myasthenic reaction, and since it was first described by Jolly it has been found to be present in the majority of the cases in which the examination was made. Even after exhaustion to faradism a vigorous contraction is at once induced by galvanism. In no case has reaction of degeneration been demonstrated.

It is very striking that in a disease with such pronounced and characteristic motor defects, which often lead to a fatal termination, there remains throughout a complete absence of so many of the well-known signs of organic disease in both the nervous and muscular systems. There is no atrophy of the affected muscles and fibrillary twitchings are absent; the special senses are unaffected and intelligence is good to the end, though in a case reported by Collins there was rapid exhaustion of the senses of sight and hearing; there are no sensory or trophic disturbances; there is no ataxia and the pupils are normal. There are no bladder or rectal troubles and the superficial reflexes, arm and knee-jerks are normal, or present no material change; in a few cases it is said that the knee-jerks became exhausted after repeated tappings.

The course of the disease is characterized by the tendency to remissions and exacerbations already mentioned. In some instances the affection is almost limited to the bulbar muscles, but in most cases it is extensive in its distribution. The duration is indefinite; it may be chronie, as in Dreschfeld's patient who died from respiratory paralysis fifteen years after the onset of the disease, and in one of Buzzard's, who is still living eight years after the symptoms first appeared; on the other hand, it may be very rapid, as in an example reported by Widal and Marinesco, ${ }^{6}$ where death occurred fourteen days after the onset of the first symptoms.

Diagnosis.-The diagnosis must depend upon the recognition of the characteristic features of the disease, the weakness and early fatigue of the muscles, the implication of other muscles than those innervated by the bulbar nuclei, especially those of the eyes and extremities, the tendency to remissions and exacerbations and the myasthenic reaction, with normal intelligence and the absence of atrophy and fibrillary twitchings, of sensory disturbances, of bladder and rectal troubles while the reflexes are normal. It is perhaps most likely to be mistaken for hysteria, neurasthenia, true bulbar paralysis or multiple neuritis, but a careful examination associated with the history of the case should prevent one falling into error. In this connection a case was recorded by Allbutt:7 "Miss — aged 18, caught cold six months previously, followed by stiffness of the tongue and jaws; this stiffness remained for a few weeks and then disappeared. She now moves her tongue and jaws and indeed every muscle of the face, throat and orbit normally. Of late she has begun to talk oddly, 'as if with a potato in her mouth.' This gets worse as she proceeds, when her utterance becomes involved and she stops." She had difficulty in swallowing, "she choked over her meals, more when she was tired, that is, at the later meals of the day." No evidence of hysteria or other neurosis or of palsy or atrophy was found, nor any other evidence of organic disease, except that the "forefinger of the left hand seemed weak and fumbling." A diagnosis of hysteria was made and for two or three weeks she made considerable improvement. Then one evening her symptoms, especially the swallowing: grew worse again and next morning before breakfast "she suddenly fell to the ground, was convulsed, turned blue and died at her mother's feet." Such a case in the hands of so competent a diagnostician as Professor Allbutt shows how readily the true nature of the disease may be overlooked, especially when the symptoms are so apparently indefinite and so variable as in the example recorded. A discussion on the differential diagnosis between myasthenia gravis and the various diseases with which it may be confounded is hardly necessary. As a rule a careful examination of the patient will render the exclusion of other diseases which may resemble it comparatively easy.

Prognosis.-Of the 62 recorded cases, 23 terminated fatally, the average duration of life after the development of the first symptoms being about $11 / 2$ years. As in many instances the duration is protracted, and as several of the reported cases have come under notice only during the past year, it will be evident that these figures probably under-rate the true gravity of the disease. It is probable that complete recovery sometimes takes place. Attacks of dyspnea from weakness of the respiratory muscles are of the gravest possible omen, and the patient's friends should be warned that death may occur suddenly during one of these attacks.

Treatment.- No specific treatment is known. Complete rest, mental and physical, and freedom from all forms of excitement should be enjoined. Various drugi have been tried, but there is no evidence to show that any drug has a beneficial effect on the disease. Strych nin has naturally been tried, but so far without result One patient of Buzzard's made a great improvement under thyroid extract, but in another it was given without benefit. Faradism appears to have done harm, while galvanism and massage did no good. General tonics, as iron, arsenic and cod-liver oil may be of use in maintaining the general health.

\section{REPORT OF CASE BY DR. J. R. BUIST}

According to the diagnosis of Dr. E. G. Wood and myself, the case we are about to report should be put in a class of nervous affections, the prominent feature 
of which is a peculiar paralysis, or, more strictly speaking, a paretic state of many muscular groups, upon exertion rapidly showing fatigue, soon succeeded by absolute loss of power if the action of the muscles is persisted in.

It is only in the last eight or nine years that a real clinical entity has been accorded this abnormal state of the neuromuscular apparatus. As much as twenty years ago some cases first attracted the attention of medical men in Germany and England, and were reported under different designations.

The foremost neurologists of Germany, France and England are, at the present time, evincing very great interest in these cases, and studying hard to solve the hidden problems of causation and pathology.

Let us recollect that most forms of disease when first recognized, appear infrequent, but as familiarity with the clinical features increases, we find that particular cases are not so uncommon. At all events we know that this will be the first case ever described and reported in the Southern States, and the sixth ever given to the profession in America. Dr. Wood and myself are glad that this distinction will belong to the Nashville Academy of Medicine.

All the articles written on this subject unite in stating that the first reported case that came to an autopsy was that of Dr. Wilks of Guy's Hospital, published in 18\%r. The examination of the brain and spinal cord were carefully and fully conducted, and as no lesions of these structures were found, Dr. Wilks gave his case the name of "Bulbar Paralysis without Anatomical Base."

Along about the same period Erb of Germany published one or two cases. No other case was reported for ten years after. From 1887 to 1893 quite a number were reported in Germany, France and England. About that time Jolly of Berlin appears to have been the first to use the term "myasthenia gravis pseudoparalytica." In 1893 Dr. Dreschfeld published a case under the heading "Polio-encephalo-myelitis without Anatomical Foundation." In 1896, Collins s uses the term "asthenic bulbar paralysis." Since then the text-books of Church and Peterson, Allbutt's "System of Medicine," and some other recent works employ the designation of "asthenic bulbar paralysis." Dr. Buzzard of London, in a lecture last March, used the term "myastheni: gravis," and so does Dr. Sanger Brown of Chicago in the last published case.

To our minds this term "myasthenia gravis pseudoparalytica" is by far the most appropriate yet employed. Without committing us to any theoretical doctrine of its etiology and pathology: or involving us in the necessity of localizing the disease, it simply gives us the most conspicuous clinical features.

Mr. E. R. R., white, 34 years old, a native of Giles County, Tennessec, and a resident of Carthage, Tean. came under our care on Nov. 23, 1900.

Family History. - His father is said to have been an active and healthy man, and to have died at the age of 72 , in an apoplectic stroke. Seven years previously he had a slight attack of hemiplegia, which was recovered from, except that the facial paralysis persisted. He had abdominal dropsy a year before his death. His mother was a woman of good constitution and died at the age of 73 , of pleurisy. There were ten children, two by a first marriage and eight by the secondthe patient is the youngest. His brothers and sisters seem to have been sound, of strong constitutions, except one brother now 48 years old, who, when 18 years of age, had an attack of typhoid fever. Ever since he has had tremulous movements of his hands. He can not write well; his condition has not changed in thirty years. One sister, now 38, with three children, after the birth of her last child-eight years ago-seems to have had puerperal mania. Her mind remains unsound, but she is not in an asylum.

Personal History.-The patient is a man of good intelligence and fair education. He has lived mostly in Tennessee, a few years in Florida, and has traveled abroad. He has always enjoyed excellent general health, usually weighing 140 pounds. He has led a very active and sometimes laborious life, being engaged in varicus mercantile pursuits, has had all the ordinary diseases of childhood, and a slight attack of influenza when 20 years old. This left no serious sequelæ.

Ten years ago, while working very hard in a wholesale drugstore, he suffered with lumbago, which confined him to bed for two weeks, and two years later he began to have pains in both legs and feet, with much restlessness of the latter at night. This condition lasted, with intermissions and in decreasing severity, up to two or three years ago.

He went to Europe in the summer of 1899, on business and for travel, remaining there until April, 1900. While in London, during February, drooping of the left eyelid occurred, and first attracted his attention while playing billiards-he was annoyed by the eyelashes coming in the field of vision. This drooping increased so that in two weeks complete ptosis of that lid existed, and he consulted an oculist. After three weeks partial recovery occurred and he has remained about the same ever since. He can not fix the date of the drooping in the right eye, but is sure that it was some months subsequent to the occurrence in the left. He had no diplopia at this time.

On April 20 he first observed that at times he saw double, and then visited another oculist in London. After examination he was advised to return home, as his case looked suspicious of serious trouble. He arrived in New York on May 10 , and consulted an oculist for diplopia. He was wearing a shade over the left eye at that time, to avoid double vision. He reached this eity on the way to Carthage on June 1, and walked from one train to another, in the station, carrying two heavy satchels. On taking hold of the hand-rail with his left. hand, he found he had lost power in the arm and could not lift. himself up by it. This was the first weakness he had experienced in any of the extremities. On June 10 he again noticed the weakness of the left arm when he tried to lift his baby. About June 15, while at home. he awoke from sleep in the middle of the night with very peculiar feelings - there was a wave of tingling passing through his body, great oppression over the chest and stomach, and he thought he was about to die. He had not caten imprudently, but a dose of bicarbonate of soda gave him relief, and he was better before the doctor arrived; it was thought likely he had had indigestion. He says it was not at all like night-mare. Subsequently, at long intervals, he had slight recurrences of this.

Early in July he first found he had lost power in the right arm-discovering this by his inability to put this hand into his hip pocket. Two weeks iater, in going into the village one-fourth of a mile from his home, he took a short-cut and had to cross a stile steps, so he reached out both hands and sought the fence to help him up, but his hold gave way and he fell backward to the ground. He recovered in a few minute: sufficiently to walk a few hundred yards to a doctor's office. He was then in a state of great prostration-pulse from 110 to 120 and weak. He had to be taken home in a carriage.

A few days after this he went to Red Boiling Springs and remained there two weeks. Just before leaving home, he thinks his lower limbs were weak. His stomach and digestion had become disordered and he felt badly and was losing flesh. He made no improvement at the Springs, and in a few days after returning home, started to Battle Creek Sanitarium in Michigan. By September 15 his muscular weakness had in. creased so much that he was unable to raise himself in bed or to dress himself unaided.

He remained at this institution from August 1 to October 1, receiving the usual treatment of massage, hot and cold packs, electricity, etc., the paretic state all the while increasing, although he was much relieved of his gastric disturbance.** 
Through October, and up to November 21 , he remained at home, during which time he thinks his condition grew no worse. He has never had the least trouble with his bladder. His urine has been repeatedly analyzed and nothing abnormal found. His bowels are usually good; no rectal paresis. He never had headaches, no febrile attacks and no pains except as above noted.

EXAMINATION OF PATIENT BY DRS. WOOD, J. R. AND W. E. BUIST; AT 8 P. M. NOVHamer 23 , AND 8 A. M. NOVEMBER 24 .

$\mathrm{He}$ was fairly well nourished, color good, intelligence excellent, memory good. His forehead showed transverse wrinkles distinct. His expression was calm, the lower half of his face smooth and symmetrical, both at rest and in action. There was partial double ptosis. When his eyes were tixed on any object held above the eyes, the upper lids would gradually fall until the eyes were almost elosed. Both eyes could be tightly closed.

Ophthalmoplegia (Partial).-The movements of both eyeballs were defective in all directions-weakest in the left external rectus. Convergence was fair. On turning the eyes to their limit in any directión. oscillatory movements soon appeared. The pupils were normal in size and shape, the reaction to light and accommodation good. The fundus was normal in both eyes. There was no facial paralysis, but the lips were weak and easily fatigued on suction. The tongue protruded in a straight line and with normal force, but soon began to quiver and in less than two minutes would fall back. The palatereflex was normal, and moved well in both sides. The jaw-jerk was active, but on biting the temporals and masseters were weak and soon became fatigued; eating, swallowing and talking tired him in a few minutes. The neck muscles were sound and the trapezii quite active and strong. The pectorals and serrati were weakened, the latissimi good.

Arms. - The grip was very fecble in both hands. The dy. namometer showed right 25 and left $2 \pi$, and was better at the morning examination-32 and 35 . Flexion of the elbow was active and strong if not resisted, but weak against resistance. The deltoids were flattened and small. There was no atrophy of the infra and supraspinati. He could raise his arms, Hexed at the elbow, so that the fingers reached the top of the ears, and could maintain them, the right 25 seconds, the left 20 , and then they would fall down. He could not put his hands in his hip pocket. His chest and shoulders were symmetricalno fibrillary twitchings. Chest expansion: Expiration, $301 / 2$ inches; inspiration, $221 / 2$. After eight or ten deep inspirations, the power of expansion diminished to three-quarters less. The patient could write for a few minutes, but the handwriting then rapidly changed, becoming feeble, with irregular, illformed letters.

Lower Limbs. - These were weak generally, but with no atrophy. The thighs measured the same, and the calves were equal. In the recumbent posture he could put one leg over the other, and sitting could, with the greatest difficulty, throw one knee over the other-he could flex and extend the knees easily and equally. He could not raise either limb against resistance. When lying down he could not rise up unassisted, and when sitting could not rise to the erect posture without help. He could stand erect alone. Romberg's symptom was absent. Walking twice across the room his gait was good, his step being a little quicker than it should be, but after the third round his limbs got tired and he had to support himself or he would have fallen. The knee-jerks were very active when lying on his back and rather more than normal when sitting, but equal in both limbs. There was no foot clonus, and no extensor response of the big toes.

Myasthenic Reaction.-Tested at 8 a. m., November 24, the biceps responded well the first half minute, then grew weak, and responses ceased in about three minutes. Responses of the deltoids were very weak, and became exhausted in $2 \frac{1}{2}$ minutes. Both arms were alike.

Sensation.-There was no pain anywhere. Cutaneous sensibility was everywhere unimpaired to touch and pain-temperature test not used. The muscular sense was good. He

* A letter from Battle Creek, Dated Nov. 12 (received Dec. 10), states that "his case is unique, and called myasthenia gravis." could touch the point of his nose with a finger of either hand with his eyes shut, without tremor. No ataxic gait and no ataxic movement of arms existed. The organic reflexes were normal, and sexual power good. The symptoms of weakness and fatigability of the muscles were more pronounced in the evening examination than on the following morning.

The patient was under observation and treatment until December 11, and then went home. During this time the pulse and temperature remained normal, that is, between 76 and 88 and 98.6 to 99 respectively. His sleep was usually good and undisturbed. His appetite was fairly good and bowels regular. The urine was found normal in quantity and quality. His knee reflexes wcre frequently examined, almost always active and, when not so, hand reinforcement would develop them to fully normal. He wrote two short letters, the first he had written in some time; after the first page the hand-writing became bad. His digestion seemed easily disturbed and he was afraid to eat many articles of ordinary diet. On the evening of November 29 , in less than an hour after dinner, he was seized with a tightness and oppression of the chest, some difficulty in swallowing and a sense of general prostration. His chest expansion was slight, even with effort, and his facial expression anxious. There was slight nausea when the head was raised a little, but no vomiting nor any epigastric pains or uneasiness. His voice was weak, and tired easily. This condition was recovered from in a few hours. He said he had felt such attacks before but lighter in degree. On another occasion he found slight difficulty in deglutition, the milk getting up in the posterior nares. The muscles were always a little stronger in the mornings, especially the levators of the eyelids. He was generally in a good humor, hopeful and patient. No twitchings nor tremors were ever present.

He improved in muscular power perceptibly while under our observation, the grip of the right hand 35 . of the left 36 , at the time he left. He did not improve any in flesh or general condition. He could walk across the room eight times without giving out. Dr. L. B. Graddy was kind enough to examine the eyes on the day he left, and found the pupils normal. The fundi in both globes were equally normal, vision normal, and the vocal cords acted normally.

Treatment.-Complete rest in bed was ordered. The thyroid gland was tried a few days but abandoned, because it seemed to produce headache. Cod-liver oil and phosphorus was given for a few days, but his stomach rather rebelled and he was placed on codein, $1 / 8$ gr. every three or four hours, which he still takes tentatively.

\section{COMMENT.}

Making a summary of the symptoms presented in the above record, we have the following clinical picture: A young man, previously healthy, with no antecedent illness and no irregular habits, is taken with a gradually. advancing loss of power in the muscles innervated by the third nerve; then marked improvement up to a certain point. After a few months he suddenly becomes aware of a partial loss of power in the left arm. This slowly increases, when unexpectedly his lower extremities get quickly tired, and one day in attempting to get up a stile steps he staggers and falls back. Both arms and both legs now rapidly lose power, so that on very slight exertion complete exhaustion of voluntary power takes place. Meanwhile no change in sensibility and no impairment of mind, no abnormal condition of reflexes, and no disturbance in the circulatory system have occurred. The paretic muscles, though diminished in size, do not show real atrophy, and respond to electric stimuli. This irregularly progressive march of paresis finally invades the muscles of mastication, deglutition and respiration. This, it seems, is essentially different from the clinical history and behavior of any other form of neurotic disorder.

If we compare the above symptom-complex with the features of those nerrous affections which most resemble 
it, and with which it has been most frequently contounded, we can easily differentiate "myasthenia gravis" from each and all.

REFEREXCES.

1. Buzzard: British Med. Jour., Mareh 3, 1900.

2. Shaw : Brain, 1890.

3. Jolly : Berliner klin. Woch., $189 \overline{5}$

4. Buzzard: Brain, 1900

5. Suckling: British Med. Jour., 1893.

6. Widal and Marinesco: La Presse Med., April 14, 1897

7. Allbutt: System of Medicine.

8. 'Twentieth Century Practice.

9. Ireschfield: Brit. Med. Jour., 1893, Vol. ii, p. 177.

LIMITATIONS OF THE LARYNGOLOGIST IN THE GENERAL TREATMENT OF NOSE AND THROAT DISEASES.

H. W. LOEB, A.M., M.D.

ST. LoLIS.

The tendency observable in modern laryngologic literature, to ascribe to the general system a closer etiologic relation with the nose and throat, must have one important effect-to call for a more general treatment of the upper respiratory tract.

Before the local propaganda had practically absorbed the attention of specialists in our line, the general or systemic means comprised in great measure the plan of treatment of nose and throat diseases. And now that localism has had full sway, it is to be expected that the general treatment should come more into vogue. Perhaps, if it were merely a return to former conditions, there would not be much call for a paper on limitations in this regard. But we must not forget that during these years changes have taken place which have increased our knowledge tenfold and our range of instrumentation and manipulation a hundredfold; which have so added to the science of medicine that no man can encompass it; which have so multiplied the fields of work that division of labor is not alone a pleasure but a necessity. Knowledge is no longer cloistered in the minds of a few men; it is open and free to him who will but learn; and its adherents are no longer numbered by the few but by the many. Naturally, this encourages competition in the acquirement of knowledge and struggle for supremacy in the application of knowledge to the various fields of human activity.

The pace set by all these workers results in the development of the special lines and to such an extent that he who would keep up to the standard set by these pacemakers must confine himself, almost exclusively, to the domain of his own work. Hence, when there is a call to return to a general view of medicine, for the cure of certain nose and throat affections, the specialist who has been in great part a localist finds himself, if he be conscientious to patients, in a field in which he can not work, except to the detriment of his special duties, which ean not receive the attention they require.

It is a consideration such as this that has led me to present here some points in the relation of the throat and nose to the general system, which seem, at least, to substantiate the proposition that has been made to the effect that the practitioner and not the specialist should conduct the general treatment. I know the argument is advanced that the specialist should be first a general practitioner, and then become a specialist, that he should never sacrifice his interest in general medicine for the detail of his specialty. And yet the progress in general medicine is so great that ten years make a

* Read by Title to the Section on Laryngology and Otology, at the Fifty-first Annual Meeting of the American Medical Association held at Atlantic City, N. J., June 5-8, 1900. tremendous difference and the specialist, after such a lapse of time from participation in the actual practice of general medicine, will find such a change that he will be in almost an unknown world. Then, he can barely keep up with the literature of his own line-not to speak of the tremendous amount that is to be included under the head of general medicine. Every month there are published about 300 articles devoted exclusively to the nose, throat and ear-the whole domain of medicine includes many thousands. Which is to be chosen, the relatively few or the impossible many? Another thing, the laryngologist's attention is, in the main, devoted to manipulation with instruments of examination and of therapeutics-can he be expected to be proficient in these and at the same time capable of outlining the plan of treatment which comprises conditions and circumstances remote from the nose and throat?

Because a paralyzed left vocal band is due to pressure upon the left recurrent, which in its turn depends upon. a tubercular pleurisy or lymph node, is that any reason why the laryngologist should treat the tuberculosis? It seems to me that his function was completed when he discovered and localized the paralysis. Because chronic inflammations of the larynx and pharynx are often associated with bronchial and pulmonary conditions, and because the heart is in anatomic relation with the lungs and bronchi, is that any reason why the laryngologist should conduct the treatment of heart affections, or perchance kidney affections, which cause or result from cardiac diseases? I can understand how one who devotes his attention to the heart and lungs must be somewhat proficient in the diagnosis--not treatmentof nose and throat affections, but that the laryngologist should affect to include the lungs, heart and kidney is either presumptuous or evidence of a lack of full interest in his own line. It must be admitted, however, that circumstance sometimes throws a laryngologist into the necessity of including diseases of the lungs in his work, but few are equal to the task of properly filling both functions.

I say nothing of the justice which should be accorded the general practitioner who sends patients to laryngologists and to other specialists, for that matter, for his proper territory should not be encroached upon by the very men to whom he refers his cases.

Another phase of the question is to be found in the justice to patients, which we all should and do accord. Should we whose view is largely limited by the requirement of our special study and duties assume to meet the general indications with a skill equal to the general practitioner whose efforts are continually directed toward general conditions which we are considering? Is it not better for each to fulfil his own part of the medi. cal and surgical management of the case-does not our own obligations to our patients demand this? We can, naturally, advise and consult with the general practitioner upon these matters, but to carry out these general indications is surely his particular function.

Relative to the bearing which general medicine and other specialties have upon laryngology, it will be interesting to consider the various conditions which may be associated with hemorrhage of the upper respiratory tract. According to Hagedorn, they include the following: Changes in the blood-vessels and blood itself, hemophilia, morbus maculosus Werlhofii, scorbutus, purpura hemorrhagica, phosphorus poisoning, anemia, leukemia, pseudoleukemia, diabetes mellitus, fatty degeneration of the blood-vessels, diphtheria, variola, ileotyphus, pyemic processes, gout, influenza, acute rheumatism, vicarious: 